\title{
Hernia de Garengeot complicada con absceso inguinal, revisión de la literatura
}

\section{Garengeot's hernia complicated with inguinal abscess, literature review}

\author{
Francisco Xavier Cabrera-Mendoza, ${ }^{*}$ Aurelio Barrera-González, \\ Jesús Galindo-Jiménez, ${ }^{*}$ Joel Castillo-Espinoza, ${ }^{\ddagger}$ Edgar Cantú-Rodríguez ${ }^{\ddagger}$
}

\section{RESUMEN}

Se conoce como hernia de Garengeot al deslizamiento del apéndice cecal a través del canal femoral, su presentación complicada con absceso es en extremo rara y causa de confusión diagnóstica. Se describe un caso que presenta absceso inguinal resultado de un cuadro apendicular agudo en una hernia de Garengeot (y el abordaje diagnóstico y terapéutico que llevó a cabo). La revisión de casos aislados coincide en que se realice apendicectomía, así como hernioplastía de acuerdo con el dominio y experiencia del cirujano.

\section{ABSTRACT}

Sliding of cecal appendix into femoral canal is known as Garengeot's hernia, its complicated presentation is extremely rare, and of difficult diagnosis. We describe a case presented with inguinal abscess resulting from an acute appendicitis in a Garengeot's hernia (and its diagnostic and therapeutic approach). Intense revision of isolated cases around de world agree in realize appendectomy and crural defect hernioplasty according to skills and experience of the surgeon.

\section{INTRODUCCIÓN}

$\mathrm{E}^{\mid}$ I deslizamiento del apéndice cecal en un saco herniario inguinal es conocido como hernia de Amyand, descrito inicialmente por Claudius Amyand, quien realizó simultáneamente la primera apendicectomía. ${ }^{1}$ La hernia de Garengeot, reconocida así por el cirujano homónimo francés que describió un cuadro apendicular agudo en una hernia femoral en 1731 , se ha asociado con defectos congénitos, por lo cual es mucho más frecuente en mujeres. El apéndice cecal es un hallazgo raro en hernias femorales y aún más rara la presencia de apendicitis aguda dentro del canal femoral, representado cerca de $0.1 \%$ de las hernias femorales $^{2}$ y de 0.13 a $0.8 \%$ de todos los casos de apendicitis aguda. ${ }^{3}$ En el presente caso se propone un abordaje diagnóstico y terapéutico para esta rara presentación.

\section{PRESENTACIÓN DEL CASO}

Mujer de 84 años, con antecedentes de hipertensión arterial sistémica y fibrilación auricular crónica en control con enalapril y verapamilo respectivamente. Dentro de su historial quirúrgico, cuatro años previo al padecimiento actual fue intervenida de urgencia por hernia inguinal directa estrangulada derecha, con contenido intestinal, abordada en región inguinal, requiriendo resección intestinal y entero-entero anastomosis término-terminal y hernioplastía Shouldice. Su postoperatorio satisfactorio, siendo egresada sin complicaciones. Su padecimiento actual inicia con masa en región inguinal, la cual crecía lentamente, presenta dolor inicialmente sordo, leve y no le da importancia; también crecimiento importante en las siguientes dos semanas, notando cambios de coloración, eritema y dolor importante, por lo

Citar como: Cabrera-Mendoza FX, Barrera-González A, Galindo-Jiménez J, Castillo-Espinoza J, Cantú-Rodríguez E. Hernia de Garengeot complicada con absceso inguinal, revisión de la literatura. Cir Gen. 2020; 42 (4): 326-329. https://dx.doi. org/10.35366/101404 
que acude a hospital general donde se sospecha nuevamente hernia inguinal estrangulada. Se realiza ecografía en región inguinal, la cual reporta aparente hernia inguinal y abundante gas, avascular en la función Doppler. Deciden enviar a nuestra institución para su manejo. A la exploración se observa agitada, taquicárdica (105 latidos por minuto), moderadamente deshidratada; se explora masa rígida, eritematosa, aumento de calor local en región inguinal, la cual desplaza a cicatriz quirúrgica previa, muy doloroso a la palpación; presenta leucocitosis de 19,250 células/ $\mu$ (neutrofilia 88.90\%), aumento en tiempo de protrombina (Tp) y tiempo parcial de tromboplastina (TpT), el resto dentro

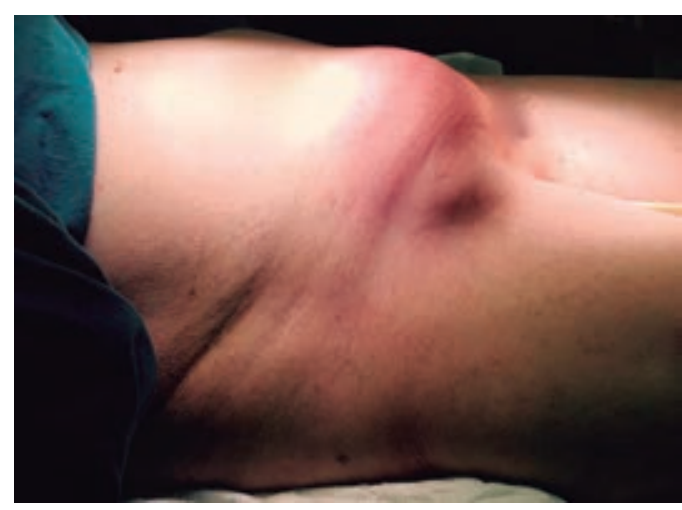

Figura 1: Presentación del paciente al llegar a urgencias.
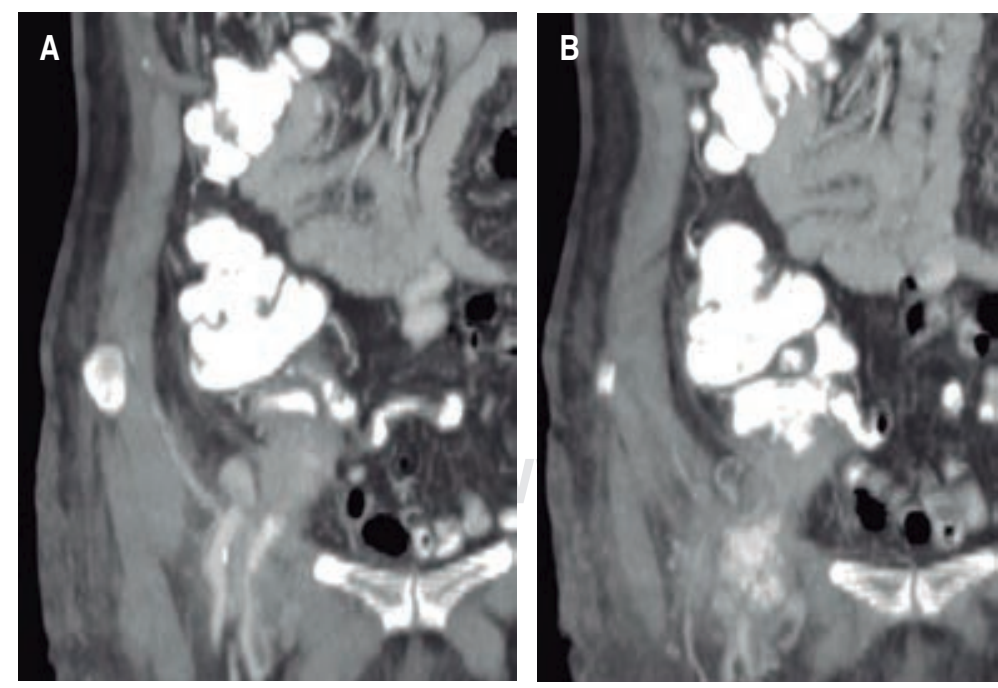

Figura 2: Imágenes tomográficas del apéndice cecal a través de canal femoral (A) y cubriendo con contraste canal femoral y colección residual (B). de parámetros normales. Se sospecha hernia inguinal recidivante con contenido intestinal, aunque la apariencia de la masa recordaba a la apariencia de un absceso (Figura 1). Se decide su paso a quirófano, bajo anestesia regional se realizó acceso por cicatriz previa, encontrando absceso importante aproximadamente $400 \mathrm{~cm}^{3}$ que se drena en su totalidad, se observa plastía inguinal previa intacta, no se observan sacos herniarios, no se observa tampoco un evidente origen de material purulento, no se puede determinar claramente el origen del absceso. Se decide manejo de herida abierta para cierre por segunda intención y continuar estudiando a la paciente. Se realizan curaciones a necesidad del gasto y se realiza horas después una tomografía contrastada abdominal a determinar sitios probables de origen del absceso, se sospecha en enfermedad diverticular complicada. El reporte por el servicio de imagen determina apéndice vermiforme edematoso, el cual sale aparentemente por el conducto femoral y una colección pequeña periapendicular (Figura 2). Se programa nueva laparotomía exploradora encontrando múltiples adherencias alrededor del sitio de anastomosis realizada hace cuatro años, las cuales se liberan alcanzando ciego y apéndice vermiforme (Figura 3). Se realiza apendicectomía con manejo del muñón Pouchet, al extraer la pieza quirúrgica se encuentra la punta totalmente perforada; el cierre del defecto herniario se hizo con dos puntos simples de polipropileno 00, se decide no colocar malla por el alto riesgo de infección. El postoperatorio fue satisfactorio, con disminución de leucocitosis a 9.43 células/ $\mu \mathrm{l}$ y normalización de Tp y TpT, con una estancia total de seis días, la herida inguinal granuló por segunda intención, continuando con curaciones y supervisión semanal hasta lograr cierre. El reporte histopatológico final menciona apéndice cecal con infiltración leucocitaria en mayor cantidad en su mitad distal, hipertrofia linfoide en su mitad proximal, punta perforada y negativo a malignidad (Figura 4).

\section{DISCUSIÓN}

Existen teorías acerca de la peculiaridad de estos casos, la más aceptada es la unión anormal del apéndice al ciego por una mala rotación, 

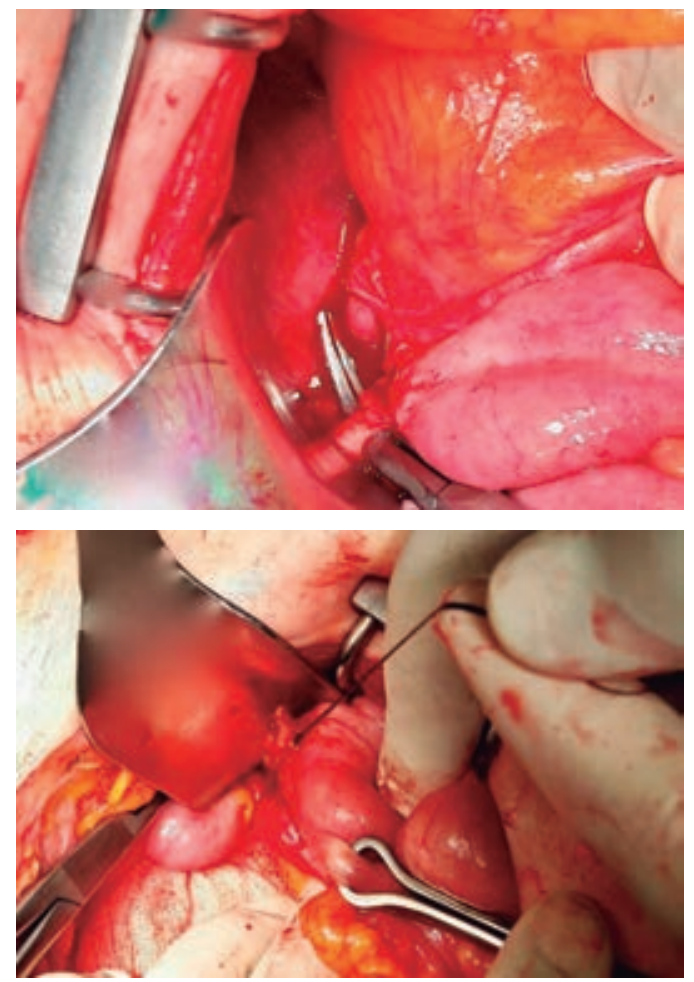

Figura 3: Apéndice cecal en hernia femoral y manejo de muñón.
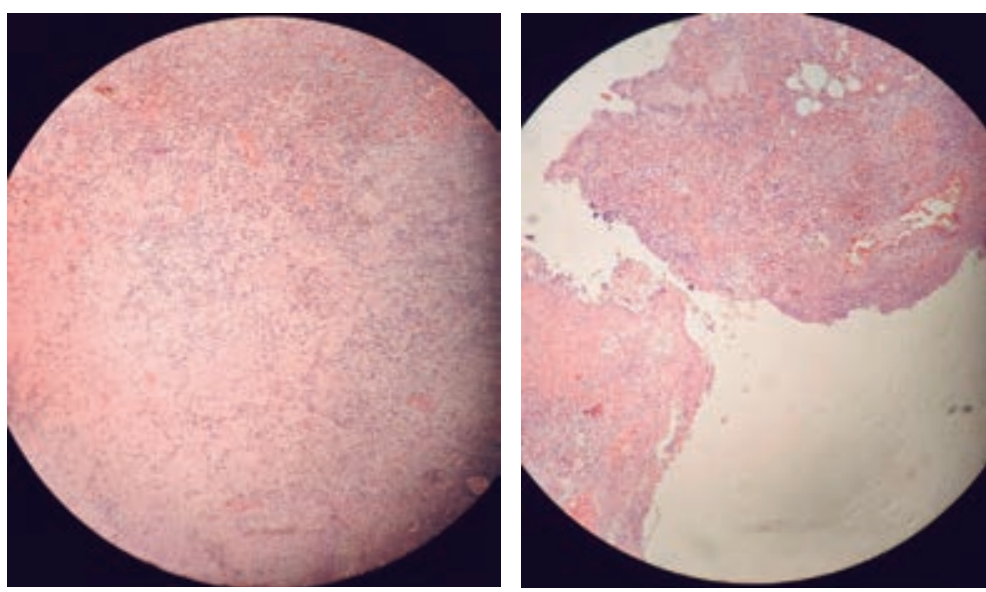

Figura 4: Microfotografias de la pieza extraida. Muestran el infiltrado leucocitario. Apendicitis fibrinopurulenta.

la cual ocasiona una localización pélvica del apéndice, con un mayor riesgo de introducirse en el canal femoral. ${ }^{3}$ La apendicitis aguda en una hernia femoral puede ser consecuencia de la estrangulación del apéndice cecal a su paso por el canal femoral, más que por la hiperplasia linfoide o presencia de apendicolito, lo que se ve evidenciado en la literatura con predominio de presencia en adultos y ancianos de estos cuadros raros. ${ }^{4,5}$ La serie más grande reportada de casos de hernia de Garengeot fue de siete durante 16 años en el Hospital Whiston, en Reino Unido, reportado por Sharma $\mathrm{H}$ y colaboradores. Se reportan cuatro casos sin apendicitis aguda donde se realizó hernioplastía con malla, y tres casos en los cuales se realizó apendicectomía más hernioplastía sin material protésico por presentar apéndice cecal inflamado transoperatoriamente. Es de mencionar que el dolor abdominal únicamente se presentó en un caso. Al igual que en este caso, un paciente presentó evolución de dos semanas con masa inguinal creciente y dolorosa, y el mismo reporte ecográfico erróneo, así como perforación hacia el canal femoral que sólo generó un absceso de origen oculto. ${ }^{6} \mathrm{La}$ presencia de un absceso o colección purulenta extraabdominal en la región inguinal asociada a una hernia de Garengeot ha sido reportada en dos casos más aislados en la literatura mundial, en los cuales se sugiere el efecto de tapón del apéndice sobre el canal femoral y, de manera adicional, la hermeticidad de la plastía inguinal previamente realizada en nuestra paciente. El diagnóstico preoperatorio es difícil cuando existen ya complicaciones como perforación apendicular, y la posible formación de abscesos y fascitis necrotizante, formas clínicas de rápido deterioro de los pacientes que nos obligan a actuar de manera cautelosa, como el drenaje inicial del absceso y planeación quirúrgica a través de una tomografía contrastada abdominal tal y como lo sugieren los grupos expertos en defectos de pared abdominal ${ }^{7}$ para poder diferenciar el origen del material purulento, como la enfermedad diverticular ${ }^{8} y$ pancreatitis aguda. ${ }^{9}$ Ya que no se encontró un defecto herniario en la plastía previa o región femoral durante el primer evento quirúrgico, se decidió esperar a un estudio tomográfico y en una segunda exploración quirúrgica resolver la patología primaria, que en este caso resultó en la perforación apendicular a través del canal femoral. No existe un consenso sobre el manejo de las hernias de Garengeot; sin embargo, en la revisión extensa de los reportes, todos coin- 
ciden en que se realice apendicectomía, ya sea incidental o en cuadro inflamatorio agudo (como lo fue este caso), así como herniorrafia del defecto femoral, con la técnica más apropiada de acuerdo con dominio y experiencia del cirujano. ${ }^{10,11}$

\section{CONCLUSIÓN}

Las masas inguinales con características clínicas propias de un absceso deben hacer sospechar patología intraabdominal que puede llevar a un rápido deterioro clínico, sobre todo en los pacientes ancianos. Los estudios de imagen juegan un rol importante en estos casos que, en manos expertas, pueden llevar al diagnóstico preciso y una planeación quirúrgica adecuada y dirigida. ${ }^{12}$ Su presentación en el lado derecho debe hacer sospechar un cuadro apendicular agudo, ya sea en una hernia inguinal (Amyand) o en región femoral (Garengeot).

\section{REFERENCIAS}

1. Michalinos A, Moris D, Vernadakis S. Amyand's hernia: a review. Am J Surg. 2014; 207: 989-995.

2. Hernández LA, León TAM, Murillo ZA. Hernia de Garengeot. Reporte de caso y revisión de la literatura. Cir Gen. 2012; 34: 78-82.

3. Caraballoso GVJ, Cabrera RJ, Alonso DN, Santana González-Chávez A, Orea Cl. Hernia de Garengeot. A propósito de un caso. Rev Med Electrón. 2018; 40: 488-494.

4. Sharma H, Jha PK, Shekhawat NS, Memon B, Memon MA. De Garengeot hernia: an analysis of our experience. Hernia. 2007; 11: 235-238

5. Martinez-Valenzuela N, Alfonso-Alfonso C, Sosa-Martin JG. Hernia de Garengeot. Rev Cub Med Mil. 2013; 42: $110-115$

6. Armstrong O. Appendix strangulated in a femoral hernia. Hernia. 2010; 14: 225-226.
7. Kagan Coskun A, Kilbas Z, Yigit T, Simsek A, Harlak A. De Garengeot's hernia: the importance of early diagnosis and its complications. Hernia. 2012; 16: 731-733.

8. Greenberg J, Arnell TD. Diverticular abscess presenting as an incarcerated inguinal hernia. Am Surg. 2005; 71: 208-209.

9. Carner S, Tan E, Warren K, Braasch J. Pancreatic abscess. Am J Surg. 1973; 129: 426-431.

10. Kalles V, Mekras A, Mekras D, Papapanagiotou I, AlHarethee W, Sotiropoulos G, et al. De Garengeot's hernia: a comprehensive review. Hernia. 2013; 17: 177-182.

11. Nguyen ET, Komenaka IK. Strangulated femoral hernia containing a perforated appendix. Can J Surg. 2004; 47: 68-69.

12. Abdulghaffar S, Almulla M, Gupta P, Mohamed BA. CT and Ultrasound findings in a case of De Garengeot's hernia. Radiol Case Rep. 2019; 14: 704-707.

Consideraciones y responsabilidad ética: Privacidad de los datos. De acuerdo a los protocolos establecidos en el centro de trabajo de los autores, éstos declaran que han seguido los protocolos sobre la privacidad de datos de pacientes y preservado su anonimato. El consentimiento informado del paciente referido en el artículo se encuentra en poder del autor.

Financiamiento: No se recibió apoyo financiero para la realización de este trabajo.

Conflicto de intereses: Los autores declaran que no existe ningún conflicto de intereses en la realización del trabajo.

Correspondencia:

Francisco Xavier Cabrera-Mendoza

Hospital Regional Monterrey, Instituto para la Salud y Seguridad Social de los Trabajadores del Estado (ISSSTE), Monterrey, Nuevo León.

Av. Adolfo López Mateos Núm. 122,

Col. Burócratas Federales, 64380, Monterrey, Nuevo León, México. E-mail: cabrera_md@icloud.com 